\title{
Dye solution optimizing staining conditions for in vivo endocytoscopy for normal villi and superficial epithelial tumors in the duodenum
}

\author{
Kenichi Godaa ${ }^{a, b}$, Akira Dobashib, Noboru Yoshimura ${ }^{b}$, Yuko Harab ${ }^{b}$ Naoto Tamai ${ }^{b}$, Kazuki Sumiyama ${ }^{b}$, \\ Masahiro Ikegamic, Hisao Tajirid
}

Dokkyo Medical University, Tochigi; The Jikei University School of Medicine, Tokyo, Japan

\section{Abstract}

\begin{abstract}
Background Vital staining is mandatory for endocytoscopy, which enables visualization of gastrointestinal mucosa at the cellular level. This study aimed to identify a dye solution that would optimize staining conditions for in vivo endocytoscopy in the duodenum, including normal villi and superficial non-ampullary duodenal epithelial tumors (SNADETs).

Methods We performed endocytoscopy in 9 patients who had normal villi (27 sites) and 20 patients with SNADETs (20 sites). The normal sites were allocated to methylene blue (MB; 5/2.5/1\%), toluidine blue (TB; $1 / 0.5 / 0.25 \%$ ), and crystal violet (CV; $1 / 0.5 / 0.25 \%)$ staining. Based on the results of normal sites, we used $1 \% \mathrm{MB}$ or $0.5 \%$ TB for staining SNADETs. Three reviewers, including endoscopists and pathologists, evaluated and scored the endocytoscopy images (1, poor; 2, moderate; 3 , good) for general image quality and visibility of structure and nuclei. We calculated frequencies and compared the proportions of the highest score of 3 (good).
\end{abstract}

Results The majority of scores of 3 for normal villi was given to $0.5 \%$ TB (81\%), followed by $1 \%$ $\mathrm{MB}$. For SNADETs, $1 \% \mathrm{MB}$ showed significantly higher scores compared with $0.5 \% \mathrm{~TB}(\mathrm{P}=0.035)$.

Conclusion Among the dye solutions evaluated, 0.5\% TB and 1\% MB achieved the optimizing staining conditions for in vivo endocytoscopy for normal villi and SNADETs, respectively.

Keywords Endocytoscopy, adenoma, dysplasia, duodenal cancer, duodenum

Ann Gastroenterol 2019; 32 (4): 1-9

\section{Introduction}

Adenocarcinoma of the small intestine is remarkably rare (incidence 6.8 cases per million) compared with other gastrointestinal (GI) carcinomas [1]. The duodenum is the most frequent site of adenocarcinomas in the small intestine [2]. The incidence of duodenal carcinoma is increasing at almost the

Department of a Gastroenterology, Dokkyo Medical University, Tochigi (Kenichi Goda); ${ }^{b}$ Endoscopy (Kenichi Goda, Akira Dobashi, Noboru Yoshimura, Yuko Hara, Naoto Tamai, Kazuki Sumiyama); ${ }^{\text {Pathology }}$ (Masahiro Ikegami); 'Innovative Interventional Endoscopy Research (Hisao Tajiri), The Jikei University School of Medicine, Tokyo, Japan

\section{Conflict of Interest: None}

Correspondence to: Kenichi Goda, MD, PhD, Associate Professor, Department of Gastroenterology, Dokkyo Medical University, 880 Kitakobayashi, Mibu, Tochigi, 3210293, Japan,

e-mail: kengoendoscopy@hotmail.co.jp

Received 20 January 2019; accepted 26 March 2019; published online 10 May 2019

DOI: https://doi.org/10.20524/aog.2019.0382 same rate as that of colon carcinoma [3] and it has the lowest 5 -year survival rate $(<30 \%)$ compared with all other small intestinal carcinomas $[4,5]$. Diagnosing the tumor at an early stage helps improve prognosis, and early-stage tumors are most likely to be treated using radical and minimally invasive endoscopic resection [6].

Advanced endoscopy-e.g., high-definition and magnification endoscopy with image-enhancing technology such as narrow-band imaging (NBI) - facilitates the detection of superficial duodenal epithelial tumors and improves the diagnostic accuracy [7-9]. In superficial non-ampullary duodenal epithelial tumors (SNADETs), studies suggest that magnification endoscopy with NBI can differentiate low- (LGD) from high-grade dysplasia (HGD) and superficial carcinoma, indicating treatment with endoscopic resection [7-10].

Even if advanced endoscopy could provide accurate diagnosis, subsequent biopsy is required for histological diagnosis, which remains the gold standard. However, mucosal defects caused by endoscopic biopsy can lead to severe fibrosis as a result of exposure to and stimulation by pancreatic juice or bile. The severe fibrosis sometimes makes endoscopic resection 
difficult in the duodenum, especially at non-ampullary sites. Therefore, it would be desirable to evaluate histological features directly during real-time endoscopy.

Endocytoscopy (ECS) has an ultra-high magnification power (>300-fold) and can visualize microscopic structures (nuclear, cytoplasmic and glandular) at the cellular level during real-time endoscopy [11]. Studies have shown that ECS may help characterize superficial epithelial tumors in the esophagus, stomach and colon [12-19]. In the duodenum, although there are 2 studies that used ECS for the diagnosis of celiac disease [22,23], little is known about the diagnostic potential of ECS for epithelial tumors.

Topical vital staining is mandatory for cellular level observation by ECS. Previous ex vivo and in vivo studies investigated the use of appropriate dyes for ECS, such as methylene blue (MB), toluidine blue (TB), and crystal violet $(\mathrm{CV})$ in the esophagus, stomach and colon $[20,21]$. However, there has been no exploration of the appropriate dye solution regimen to optimize staining conditions for ECS in the duodenum. Therefore, we conducted a preliminary study in vivo to identify the optimal dye solutions for staining in the duodenum for ECS of normal villi and SNADETs.

\section{Patients and methods}

\section{Patients}

The present study was conducted at the Jikei University Hospital from February 2011 to April 2013. We recruited 9 and 20 patients who had an endoscopically normal duodenum and SNADETs, respectively. This study was approved by the institutional review board of the Jikei University School of Medicine (No. 22-204 6381) and conducted in accordance with the Helsinki Declaration (as revised in 2000). Written informed consent was obtained from all patients.

Patients were eligible if they met the following inclusion criteria: (i) age $\geq 20$ years; (ii) an endoscopically normal duodenum (study for normal villi); or (iii) a SNADET lesion destined for endoscopic resection (study for SNADETs). Exclusion criteria were: (i) serious underlying diseases; (ii) current pregnancy; and (iii) a recent history of chemotherapy for any malignancy. Patients with a recent history of chemotherapy were excluded because duodenal mucosal damage would preclude ECS evaluation.

\section{Endoscopy equipment, setting, and performance}

We used the prototype of ECS (GIF-Y0002; Olympus Corporation, Tokyo, Japan) connected to a video-endoscopy system (CV260, EVIS LUCERA SPECTRUM; Olympus). The GIF-Y0002 is an integrated type of endoscope with one lens that enables a progressive increase in magnification to $\times 380$ (tissue field of view, $700 \mu \mathrm{m} \times 600 \mu \mathrm{m}, 0.42 \mathrm{~mm}^{2}$ ) using a hand lever. Up to $\times 600$ ultra-high magnification is available when the electronic zoom function $(\times 1.6)$ is activated by pushing the button [24]. The consecutive zoom function allows us to find the target area through non-magnifying observation, while maintaining the center of the endoscopic view. The ECS system can visualize not only the gland duct lumens and villi, but also the shape of epithelial cells and nuclei at a focus depth of about $50 \mu \mathrm{m}$.

\section{ECS procedure}

All ECS exams were performed by a single endoscopist (NY) with ECS experience of more than 30 cases of superficial epithelial neoplasm in the upper GI tract. All patients underwent deep sedation through intravenous administration of pethidine hydrochloride (35-70 mg, Opystan ${ }^{\circledR}$; Mitsubishi Tanabe Pharma, Osaka, Japan) and flunitrazepam (0.2-0.8 mg, Rohypnol $^{\circledR}$; Chugai Pharmaceutical, Tokyo, Japan). We administered butylscopolamine bromide (20-40 mg, Buscopan ${ }^{\circledR}$; Boehringer Ingelheim $\mathrm{GmbH}$, Ingelheim, Germany) or glucagon (Glucagon G Novo ${ }^{\circledR}$; Eisai, Tokyo, Japan) as a peristalsis depressant just before ECS.

Prior to dye staining for ECS, any mucus on the region of interest was carefully flushed with water containing pronase (Pronase MS ${ }^{\oplus}$; Kaken Pharmaceutical Products Inc., Tokyo, Japan) and dimethicone (Gascon ${ }^{\oplus}$; Kissei Pharmaceutical Products Inc., Tokyo, Japan). The region of interest was sprayed with $2 \mathrm{~mL}$ dye solution and excess dye was removed by suction. The tip of an ECS scope was placed in contact with the surface and then focused.

First, the staining examination was completed in the 9 patients with normal villi. Then, based on these results, we used 2 dye solutions ( $1 \% \mathrm{MB}$ and $0.5 \% \mathrm{~TB}$ ) for the examination of the 20 patients with SNADETs.

\section{Staining examination of normal villi}

The study of normal duodenal villi used 3 clinically available staining dyes: $\mathrm{MB}, \mathrm{TB}$, and $\mathrm{CV}$. The concentration for testing was determined using $2 \times, 4 \times$ or $5 \times$ dilution of these dyes $(5 \%$, $2.5 \%$, and $1 \% \mathrm{MB} ; 1 \%, 0.5 \%$, and $0.25 \% \mathrm{~TB}$; and $1 \%, 0.5 \%$, and $0.25 \% \mathrm{CV}$ ), based on an ex vivo animal study [20].

The 9 patients with normal villi were randomly and evenly allocated 1:1:1 to 3 staining groups ( $\mathrm{MB}, \mathrm{TB}$, and $\mathrm{CV}$ ) by the envelope method (Table 1). Before staining, we chose 3 duodenal areas per patient in each of the first, second and third portions, and allocated each duodenal portion to a concentration of a dye solution. We started each staining examination from the most distal portion (i.e., the third portion) to prevent regurgitation and staining of a mucosal area with 2 or 3 concentrations of a dye solution. For instance, in a patient allocated to $\mathrm{MB}$ staining, we started staining with $1 \% \mathrm{MB}(2 \mathrm{~mL})$ and took ECS images in the third portion, followed by staining with $2.5 \% \mathrm{MB}(2 \mathrm{~mL})$ and images in the 
second portion, and finally by staining with $5 \% \mathrm{MB}(2 \mathrm{~mL})$, taking photos in the first portion.

\section{Staining examination of SNADETs}

We decided to use just 2 dye solutions for this examination because SNADETs are rare and it would be difficult to examine many dye solutions and concentrations. According to the results of the examinations of normal villi, dye solutions of $0.5 \% \mathrm{~TB}$ and $1 \% \mathrm{MB}$ yielded the best and second-best staining conditions. Furthermore, 1\% MB has been most commonly used in previous studies $[18,19,22,23]$. Therefore, $0.5 \%$ TB and $1 \% \mathrm{MB}$ were used for the staining examination of SNADETs.

The 20 patients were randomly and evenly allocated $1: 1$ to the 2 staining groups (MB and TB) by the envelope method. Ten SNADETs were stained by $1 \% \mathrm{MB}$ and 10 by $0.5 \% \mathrm{~TB}$. The staining solution was applied to the SNADET lesions with a spray catheter and excess dye was removed through suction. This staining procedure was repeated up to 3 times when the lesions could not be stained adequately.

\section{ECS findings}

Fig. 1 demonstrates typical normal villi on ECS images and microphotograph, respectively. Fig. 2 shows representative ECS images and microphotographs of SNADETs, tubulovillous and tubular adenocarcinoma in situ (Category 4.2), respectively.

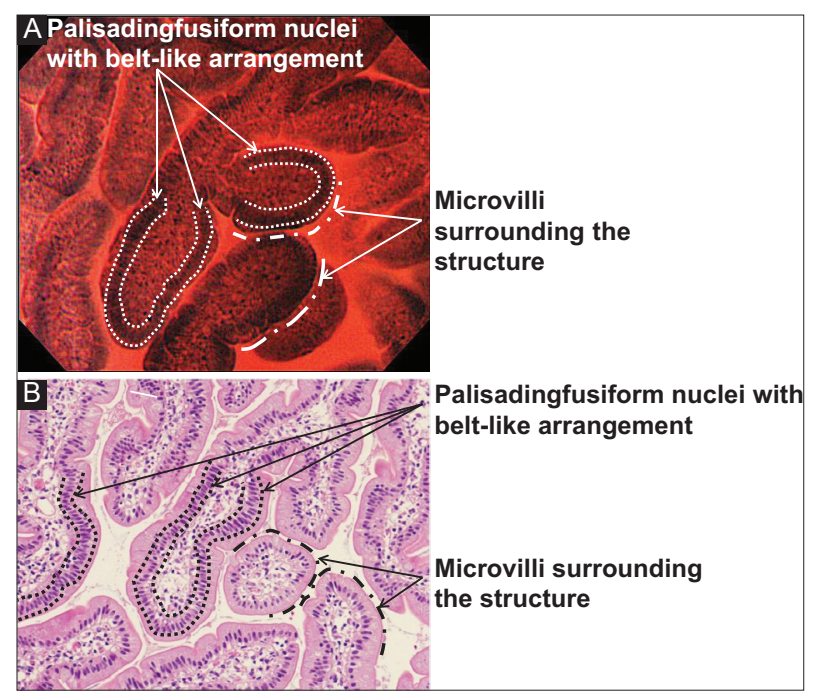

Figure 1 (A) A typical endocytoscopy (ECS) image of duodenal normal villi stained by $0.5 \%$ toluidine blue. (B) A microphotograph shows a horizontal section view of normal duodenal mucosa with hematoxylin-eosin (H\&E) staining; the 2 images are substantially identical in demonstrating villous structures covered with microvilli (transparent marginal zone in ECS and fine pinkish line in H\&E section) and foveolar epithelial cells having palisading fusiform nuclei with belt-like formation. The microvilli can be seen along with broken lines and the palisading nuclei between the dotted lines in the ECS image and microphotograph

\section{Evaluation of ECS images}

\section{Rationale for determining evaluation categories}

Quality of image is important for the precise evaluation of ECS images. Pathologists establish a histological diagnosis of noninvasive epithelial neoplasia based on nuclear and structural atypia. It is essential for ECS to visualize microstructure and nuclei. We therefore defined 3 evaluation categories as follows: general image quality, structure, and nuclei.

\section{Rating system (Fig. 3)}

Prior to the present study, 3 endoscopists (KG, NY, and AD) and one pathologist (MI) reviewed 5 ECS images of duodenal normal mucosa and 10 images of SNADETs, excluding all images from the image evaluation phase. They defined representative ECS findings of normal villi and SNADETs. Based on the representative ECS findings, they developed criteria for evaluating staining conditions.

Evaluation categories: 1. general quality, 2. structure, and 3. nuclei

Evaluation criteria and scoring method (Fig. 3)

1. "General quality" was evaluated based upon focus, optical noise, degree of staining, evenness of dyeing, and inappropriate dyeing, such as foreign matter staining.

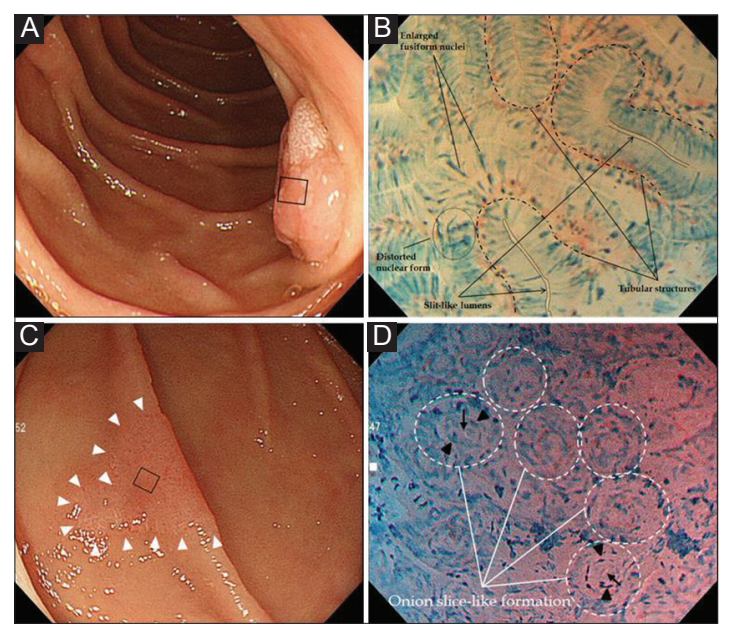

Figure 2 (A) A protruded lesion with a rough surface is seen in the second portion of the duodenum. (B) Endocytoscopy images after spraying $1 \%$ methylene blue solution for the small lesion area (black square in A). At maximum magnification, endocytoscopy visualizes irregularly-formed tubular structures with a slit-like lumen and enlarged fusiform nuclei, or distorted nuclei with a loss of polarity. Histology of an endoscopic resection specimen shows tubulovillous adenocarcinoma confined to the mucosal layer (noninvasive carcinoma, Category 4.2). (C) A slightly erythematous lesion with marginal elevation (arrowheads) is seen in the second portion. (D) An endocytoscopy image stained by $1 \%$ methylene blue solution. Maximum magnification of the small lesion area indicated by the black square in (C), a glandular structure with a small lumen (arrows) surrounded by distorted nuclei (arrowheads) appears as an onion ring-like formation. Histology of the endoscopic resection specimen showed a tubular adenocarcinoma in situ (Category 4.2) 


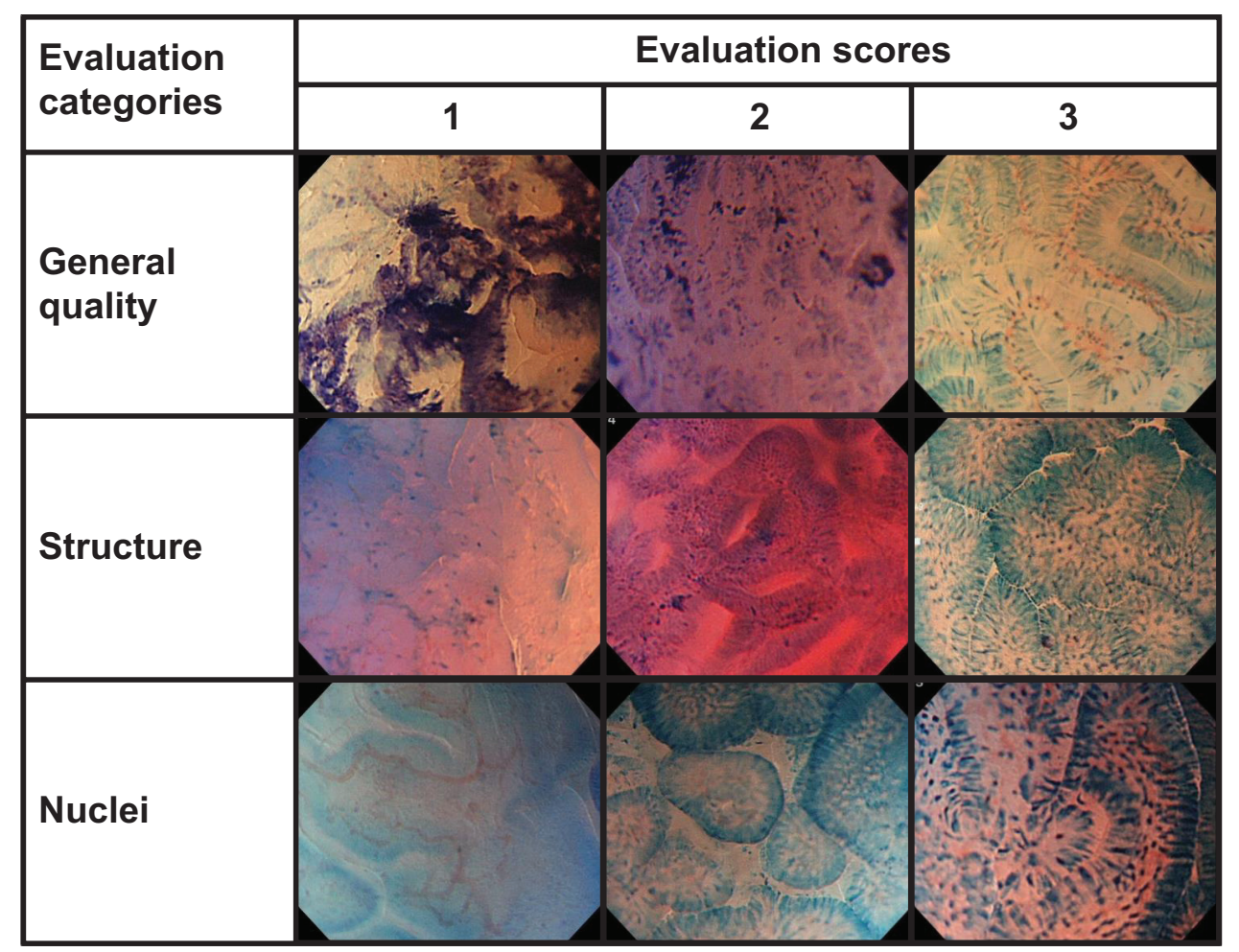

Figure 3 Representative endocytoscopy images corresponding to each score and category for reviewer's evaluation

Table 1 Patient characteristics in the staining examination of normal duodenal villi

\begin{tabular}{lcccc}
\hline Characteristic & MB $(\mathrm{n}=3)$ & $\mathrm{TB}(\mathrm{n}=3)$ & $\mathrm{CV}(\mathrm{n}=3)$ & $\mathrm{P}$-value \\
\hline Age, years, mean (SD) & $62(3.5)$ & $70(3.7)$ & $63(14)$ & $\mathrm{P}=0.15^{\dagger}$ \\
Male, $\mathrm{n}(\%)$ & $3(100)$ & $2(67)$ & $3(100)$ & $\mathrm{P}=0.25^{*}$ \\
\hline
\end{tabular}

${ }^{\dagger}$ One-way ANOVA; ${ }^{*}$ Chi-square test

$S D$, standard deviation; $M B$, methylene blue; $T B$, toluidine blue; $C V$, crystal violet; NS, not significant

2. "Structure" was evaluated based upon the visibility of cytoplasm of villi and microvilli, and abnormal structure (tubular/glandular, irregular, or disrupted pattern) in SNADET lesions.

3. "Nuclei" were evaluated based upon the visibility of palisading fusiform nuclei or punctiform nuclei in the evaluation of normal villi and abnormal nuclear shape (enlarged and/or distorted form) in SNADET lesions.

A 3-point visual analog scale for reviewers' evaluations and scoring was rated as follows: 1, "Poor" (not interpretable or invisible); 2, "Moderate"; 3, "Good" (interpretable or visible). These corresponded to evaluation scores of 1,2 , and 3 points, respectively.

\section{Preparation of image files}

The endoscopist (NY) who performed all the ECS inspections took several images of each normal duodenal site and SNADET lesion. The endoscopist created 2 image catalogs for evaluating normal villi and SNADETs as follows. The ECS images were all stored and divided into 2 image groups: a normal villi group and a SNADET group. Each of the 2-image groups was subdivided into image files corresponding to the respective staining procedure (e.g., an image file of normal villi stained by $0.5 \% \mathrm{~TB}$ ). Thus, image groups contained 27 (each dye $\times 3$ concentrations $\times 9$ patients) and 20 ( 2 dyes $\times 10$ lesions) image files for normal villi and SNADETs, respectively. Each file that included several images was numbered separately for blinded evaluation, using randomly assigned numbers from a randomization table created by a computer program (Excel 2010, Microsoft Corp., Redmond, USA). No information about the dye solution and its concentration was displayed on the images and files.

\section{Evaluation of staining conditions}

In the review of the image files of normal villi, 2 endoscopists $(\mathrm{KG}$, and $\mathrm{AD}$ ) and a pathologist $(\mathrm{MI})$ evaluated the staining conditions. In the evaluation of the image files of SNADETs, an endoscopist (KG) and a pathologist (MI) assessed the 
staining conditions. One endoscopist (AD) was excluded from reviewing the image file of SNADETs because he was considered to have inadequate knowledge of the microscopic morphologic features of SNADETs.

Three reviewers (KG, $\mathrm{AD}$, and $\mathrm{MI})$ and 2 reviewers (KG and $\mathrm{MI}$ ) assigned scores to the 3 evaluation categories of 27 (sites of normal villi) and 20 (SNADETs) ECS image files, respectively. In evaluating an image file of normal villi, each of the 3 reviewers gave an evaluation score to each of the 3 evaluation categories for each of the 3 sites stained by a dye solution. Each of the 9 dye solutions consequently received 27 evaluations in total (Table 2). Evaluation of SNADETs was more complicated than that for normal villi. Hence, the 2 expert reviewers (KG and $\mathrm{MI}$ ) mutually agreed upon and gave only one evaluation score to each of 3 evaluation categories for each of 10 SNADETs. Each of the 2 dye solutions consequently received 30 evaluations in total (Table 4).

\section{Histologic diagnosis}

Histologic diagnosis of normal ECS sites or SNADET lesions was established by an expert GI pathologist (MI) using biopsied tissues or endoscopically resected specimens. The pathologist was blinded to the information about the ECS findings. SNADET lesions were histologically graded as follows: LGD, HGD, or adenocarcinoma, corresponding to category 3 , category 4.1 , and category 4.2 to 5 , respectively, in the modified Vienna classification [25]. SNADET was defined

Table 2 Evaluation results for normal duodenal villi

\begin{tabular}{|c|c|c|c|c|}
\hline \multirow[t]{2}{*}{ Dye solutions $\left(\mathrm{n}^{*}\right)$} & \multirow[t]{2}{*}{ Evaluation categories } & \multicolumn{2}{|c|}{ Evaluation score ${ }^{\star *}$} & \multirow{2}{*}{$\begin{array}{l}\text { Frequency of the highest } \\
\text { evaluation score of } 3, \mathrm{n}(\%)\end{array}$} \\
\hline & & 1 or 2 & 3 & \\
\hline \multirow[t]{3}{*}{$1 \% \mathrm{MB}(27)$} & General quality & 3 & 6 & $19(70)$ \\
\hline & Structure & 0 & 9 & \\
\hline & Nuclei & 5 & 4 & \\
\hline \multirow[t]{3}{*}{$2.5 \% \mathrm{MB}(27)$} & General quality & 4 & 5 & $16(59)$ \\
\hline & Structure & 4 & 7 & \\
\hline & Nuclei & 5 & 4 & \\
\hline \multirow[t]{3}{*}{$5 \% \mathrm{MB}(27)$} & General quality & 5 & 4 & $16(59)$ \\
\hline & Structure & 2 & 7 & \\
\hline & Nuclei & 4 & 5 & \\
\hline \multirow[t]{3}{*}{$0.25 \% \mathrm{~TB}(27)$} & General quality & 5 & 4 & $18(67)$ \\
\hline & Structure & 2 & 7 & \\
\hline & Nuclei & 2 & 7 & \\
\hline \multirow[t]{3}{*}{$0.5 \% \mathrm{~TB}(27)$} & General quality & 2 & 7 & $22(81)$ \\
\hline & Structure & 2 & 7 & \\
\hline & Nuclei & 1 & 8 & \\
\hline \multirow[t]{3}{*}{$1 \%$ TB (27) } & General quality & 6 & 3 & $18(67)$ \\
\hline & Structure & 1 & 8 & \\
\hline & Nuclei & 2 & 7 & \\
\hline \multirow[t]{3}{*}{$0.25 \%$ CV (27) } & General quality & 9 & 0 & $6(22)$ \\
\hline & Structure & 3 & 6 & \\
\hline & Nuclei & 9 & 0 & \\
\hline \multirow[t]{3}{*}{$0.5 \%$ CV (27) } & General quality & 9 & 0 & $4(15)$ \\
\hline & Structure & 5 & 4 & \\
\hline & Nuclei & 9 & 0 & \\
\hline \multirow[t]{3}{*}{$1 \% \mathrm{CV}(27)$} & General quality & 9 & 0 & $3(8)$ \\
\hline & Structure & 6 & 3 & \\
\hline & Nuclei & 9 & 0 & \\
\hline
\end{tabular}

${ }^{*}$ Total of 3 reviewers' evaluations for 9 normal sites; ${ }^{* *}$ Frequency of each score for each category

$M B$, methylene blue; $T B$, toluidine blue; $C V$, crystal violet 
as a combination of LGD, HGD, and adenocarcinoma, in which invasion was confined to the submucosal layer [6].

\section{Statistical analysis}

We calculated the frequencies and proportions of the individual scores of evaluation categories for each dye solution. In the statistical analysis, we divided the evaluation scores into 2 groups, "1 or 2" and 3, to avoid small data pools for some evaluation categories. Quantitative parameters were compared using Student's $t$-test, while qualitative parameters were compared using Fisher's exact test or one-way ANOVA. Statistical significance was defined as a 2-tailed P-value of $<0.05$. All statistical analyses were performed using EZR (Saitama Medical Center, Jichi Medical University, Saitama, Japan), a graphical user interface for R (The R Foundation for Statistical Computing, Vienna, Austria).

\section{Results}

\section{Normal villi}

Table 1 lists the characteristics of patients. There were no significant differences among the $\mathrm{MB}, \mathrm{TB}$, and $\mathrm{CV}$ groups as regards age or sex. Typical ECS images of normal duodenal villi stained by 9 dye solutions are shown in Fig. 4 . Table 2 shows

Table 3 Comparison of evaluation results for normal duodenal villi using 3 staining dyes

\begin{tabular}{lccc}
\hline Staining dyes $\left(\mathrm{n}^{\star}\right)$ & \multicolumn{2}{c}{$\begin{array}{c}\text { Evaluation } \\
\text { score }\end{array}$} & \\
\cline { 2 - 3 } & 1 or 2 & 3 & \\
\hline MB $(81)$ & 30 & 51 & PB value ${ }^{\dagger}$ \\
TB $(81)$ & 23 & 58 & MB vs. $\mathrm{CV}: \mathrm{p}=0.18$ \\
$\mathrm{CV}(81)$ & 69 & 12 & TB vs. $\mathrm{CV}: \mathrm{p}<0.001$ \\
\hline
\end{tabular}

${ }^{\star}$ Total of 3 reviewers' evaluations for each dye; ${ }^{* *}$ Frequency of evaluation scores, 1 or 2 vs. 3 , in 3 evaluation categories (general quality, structure, nuclei)

$M B$, methylene blue; $T B$, toluidine blue; $C V$, crystal violet; ${ }^{\dagger}$ Fisher's exact test that the highest score of 3 (Good) was most frequently given to $0.5 \% \mathrm{~TB}$ for general quality of image and visibility of nuclei ( $n=7$ and 8 , respectively) and to $1 \% \mathrm{MB}$ for structure $(\mathrm{n}=9)$. Of the 3 staining regimens, $0.5 \% \mathrm{~TB}$ obtained the most top scores of 3 (Good) $(22 / 27,81 \%)$, followed by $1 \% \mathrm{MB}(19 / 27,70 \%)$. In the CV group, no reviewer gave the highest score of 3 (Good) for 2 of the evaluation categories, general quality and nuclei. $\mathrm{MB}$ and $\mathrm{TB}$ showed significantly higher scores compared with $\mathrm{CV}$ in the overall reviewer's evaluation $(\mathrm{P}<0.001$ and $\mathrm{P}<0.001$, respectively, Table 3 ).

\section{SNADET}

No significant difference in the baseline data of patients with SNADETs (age, sex, location, diameter, macroscopic type, and histology) was found between the $1 \% \mathrm{MB}$ and $0.5 \%$ TB staining groups. Table 4 indicates that $1 \% \mathrm{MB}$ achieved a significantly higher score compared with $0.5 \% \mathrm{~TB}(\mathrm{P}=0.035)$ in all evaluation categories (general quality/structure/nuclei).

No patients experienced complications or adverse reactions related to the ECS examination.

\section{Discussion}

This preliminary study was conducted to investigate the most appropriate dye solution regimen to optimize staining conditions for ECS in the duodenum. The results for normal villi showed that the highest score of 3 (Good) was most frequently given to $0.5 \% \mathrm{~TB}$, followed by $1 \% \mathrm{MB}$. In the evaluation of SNADETs, $1 \% \mathrm{MB}$ achieved a significantly higher evaluation score compared to $0.5 \% \mathrm{~TB}$.

A previous study explored an appropriate staining regimen for ECS in the esophagus, stomach and colon, but not in the duodenum [20]. Additionally, the study was mostly conducted ex vivo using porcine organs. Two studies investigated the feasibility of ECS using $1 \%$ MB to diagnose celiac disease, whereas none of the studies mentioned a reason to use 1\% MB for ECS in the duodenum. This is the first study to evaluate an appropriate regimen of dye solution for ECS in vivo for normal villi and superficial epithelial tumors in the duodenum.

$\underline{\text { Table } 4 \text { Evaluation results of } 20 \text { superficial non-ampullary duodenal epithelial tumors }}$

\begin{tabular}{|c|c|c|c|c|c|c|c|c|c|}
\hline \multirow[t]{3}{*}{ Dye solution $\left(n^{*}\right)$} & \multicolumn{8}{|c|}{ Evaluation categories and the scores } & \multirow[t]{3}{*}{ p-value } \\
\hline & \multicolumn{2}{|c|}{ General quality } & \multicolumn{2}{|c|}{ Structure } & \multicolumn{2}{|c|}{ Nuclei } & \multicolumn{2}{|c|}{ Total $^{* *}$} & \\
\hline & 1 or 2 & 3 & 1 or 2 & 3 & 1 or 2 & 3 & 1 or 2 & 3 & \\
\hline $1.0 \% \mathrm{MB}(\mathrm{n}=10)$ & 2 & 8 & 3 & 7 & 3 & 7 & 8 & 22 & 0.035 \\
\hline $0.5 \%$ TB $(n=10)$ & 6 & 4 & 4 & 6 & 7 & 3 & 17 & 13 & \\
\hline
\end{tabular}

${ }^{\star}$ No. of superficial non-ampullary duodenal epithelial tumors; ${ }^{* *}$ Frequency of evaluation scores, 1 or 2 vs. 3 , in 3 evaluation categories (general quality, structure, nuclei)

SNADET, superficial non-ampullary duodenal epithelial tumors; MB, methylene blue; TB, toluidine blue; ${ }^{\dagger}$ Fisher's exact test 


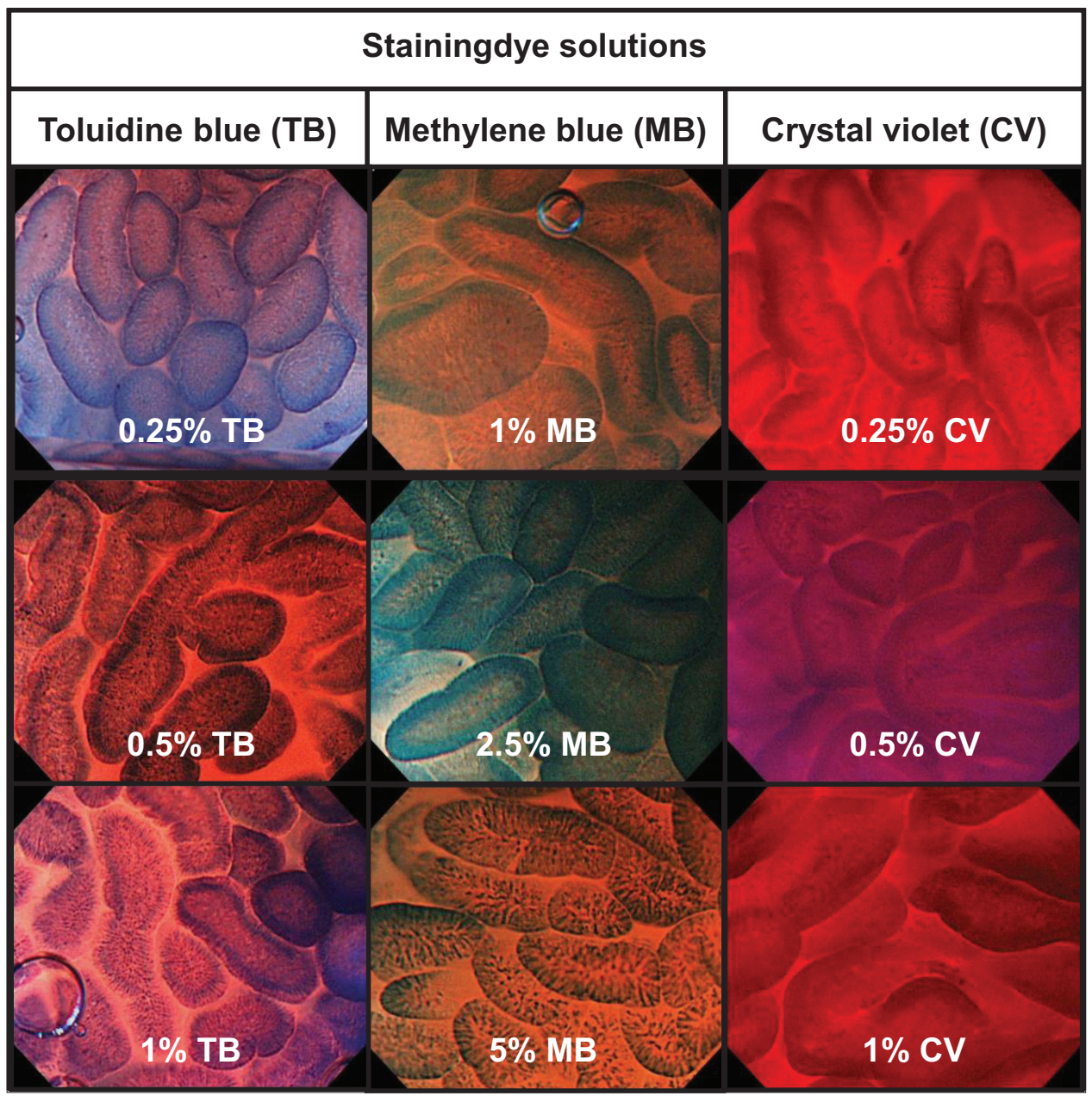

Figure 4 Typical endocytoscopy images of normal duodenal villi stained by 9 dye solutions

The previous study using porcine organs showed that the most appropriate dye was $1 \% \mathrm{MB}$ for the esophagus, and $0.25 \%$ TB for the stomach and colon [20]. Considering the lumen of the duodenum is covered with a columnar epithelium similar to the stomach and colon, it seems reasonable that $\mathrm{TB}$ received the highest evaluation score for normal villi in this study. The best concentrations of TB in this study were higher than those in the previous ex vivo study using resected porcine specimen $(0.5 \%$ vs. $0.25 \%)$. We deduced that the different concentrations would be associated with different part of the digestive tracts (duodenum vs. stomach and colon) and their mucous secretions, which would have been influenced more directly under in vivo than under ex vivo staining.

As in the previous study [20], this study demonstrated that $\mathrm{CV}$ was considerably inferior to $\mathrm{MB}$ and $\mathrm{TB}$ for staining normal villi adequately, especially as regards the visibility of nuclei. Although the reason is not clear, CV cannot be used as a staining dye for optimizing ECS observation in the duodenum, in contrast to ECS studies that used a doublestaining technique ( $\mathrm{CV}$ and $\mathrm{MB}$ ) in $\mathrm{GI}$ tracts other than the duodenum [14-16,21]. Further studies are needed to investigate which double-staining technique $(\mathrm{CV}$ and $\mathrm{MB}$ versus $\mathrm{MB}$ and $\mathrm{TB}$ ) is more appropriate for optimizing ECS observation in the duodenum.

An experimental study showed that the damage to DNA is increased in Barrett's mucosa after chromoendoscopy with $0.5 \% \mathrm{MB}$, and that the effect was dependent on the presence of both $\mathrm{MB}$ and the white light illumination of the endoscope [26]. The results suggested that exposure of Barrett's mucosa to DNA damage during endoscopy warrants caution since it could accelerate carcinogenesis. This adverse effect of $\mathrm{MB}$ staining seems to be speculative and has not been validated in living cells other than Barrett's esophagus. No study has shown DNA damage related to the use of TB and CV for vital staining in GI tracts. Further studies may be needed to explore the lowest concentration of dye solution needed to stain the tissue adequately, or to develop novel techniques that do not require the use of dye.

As with ECS, confocal laser endomicroscopy (CLE) can offer real-time in vivo observation of living cells during conventional endoscopic observation [27,28]. Intravenous fluorescein is usually used for CLE to contrast cellular structures, but fluorescein does not stain nuclei. ECS may have an advantage in allowing the observation of both cellular and 
structural atypia in neoplastic lesions, because the histological diagnosis of neoplasia is established depending on the degree of cytological and structural atypia. A prospective randomized non-inferiority trial indicated that ECS was non-inferior to standard biopsy for the discrimination of colorectal neoplastic lesions [29].

The results of this study provide valuable information about an appropriate dye solution for optimizing the ECS observation of normal villi and epithelial tumors, because no previous study explored a suitable dye solution or its concentration for ECS in the duodenum. Several limitations should, however, be acknowledged. First, this is a preliminary single-center study implemented by a small number of reviewers and patients, even though SNADETs are rare. Further definitive, fully powered multicenter studies are warranted to validate these study results. Second, ECS images were evaluated in an offline manner using still images rather than real-time images. Although real-time evaluation is important, we used still images so that the reviewers' evaluation could be made in an unbiased manner (i.e., reviewers were blinded to the kind of dye used). Third, this study did not measure the times required to adequately stain normal villi or SNADETs. A further in vivo study to determine the staining time is needed, because it would affect the estimation of the application of ECS for practical endoscopy.

In conclusion, the findings of this preliminary study suggest that $0.5 \% \mathrm{~TB}$ and $1 \% \mathrm{MB}$ are appropriate dye solutions to optimize staining conditions for the ECS observation of normal villi and SNADETs, respectively. A further in vivo ECS study is needed to validate the suggestion.

\section{Summary Box}

\section{What is already known:}

- Although advanced endoscopy facilitates the detection and diagnsis of superficial duodenal epithelial tumors, subsequent biopsy is required for a histological diagnosis, which remains the gold standard

- Endocytoscopy (ECS) has an ultra-high magnification power at a cellular level and topical vital staining is mandatory for ECS observation

- Little is known about an appropriate regimen of dye solution to optimize the staining conditions for ECS in the duodenum

\section{What the new findings are:}

- This study suggests that $0.5 \% \mathrm{~TB}$ and $1 \% \mathrm{MB}$ are the most appropriate dye solutions to optimize staining conditions for ECS observation of normal villi and non-ampullary duodenal epithelial tumors, respectively

\section{References}

1. Schottenfeld D, Beebe-Dimmer JL, Vigneau FD. The epidemiology and pathogenesis of neoplasia in the small intestine. Ann Epidemiol 2009;19:58-69.

2. Dabaja BS, Suki D, Pro B, Bonnen M, Ajani J. Adenocarcinoma of the small bowel: presentation, prognostic factors, and outcome of 217 patients. Cancer 2004;101:518-526.

3. Chow JS, Chen CC, Ahsan H, Neugut AI. A population-based study of the incidence of malignant small bowel tumours: SEER, 1973-1990. Int J Epidemiol 1996;25:722-728.

4. Zar N, Holmberg L, Wilander E, Rastad J. Survival in small intestinal adenocarcinoma. Eur J Cancer 1996;32A:2114-2119.

5. Howe JR, Karnell LH, Menck HR, Scott-Conner C. The American College of Surgeons Commission on Cancer and the American Cancer Society. Adenocarcinoma of the small bowel: review of the National Cancer Data Base, 1985-1995. Cancer 1999;86:2693-2706.

6. Goda K, Kikuchi D, Yamamoto Y, et al. Endoscopic diagnosis of superficial non-ampullary duodenal epithelial tumors in Japan: Multicenter case series. Dig Endosc 2014;26(Suppl 2):23-29.

7. Yoshimura N, Goda K, Tajiri H, Ikegami M, Nakayoshi T, Kaise M. Endoscopic features of nonampullary duodenal tumors with narrow-band imaging. Hepatogastroenterology 2010;57:462-467.

8. Lopez-Ceron M, van den Broek FJ, Mathus-Vliegen EM, et al. The role of high-resolution endoscopy and narrow-band imaging in the evaluation of upper GI neoplasia in familial adenomatous polyposis. Gastrointest Endosc 2013;77:542-550.

9. Kikuchi D, Hoteya S, Iizuka T, Kimura R, Kaise M. Diagnostic algorithm of magnifying endoscopy with narrow band imaging for superficial non-ampullary duodenal epithelial tumors. Dig Endosc 2014;26(Suppl 2):16-22.

10. Shepherd NA, Carr NJ, Howe JR, Noffsinger AE, Warren BF. Carcinoma of the small intestine. In: Bosman FT, Jaffe ES, Lakhani SR, Ohgaki H (eds). World Health Organization Classification of Tumours of the Digestive System. Lyon: IARC Press; 2010, pp. 95-101.

11. Inoue $\mathrm{H}$, Sasajima K, Kaga M, et al. Endoscopic in vivo evaluation of tissue atypia in the esophagus using a newly designed integrated endocytoscope: a pilot trial. Endoscopy 2006;38:891-895.

12. Fujishiro M, Takubo K, Saito Y, et al. Potential and present limitation of endocytoscopy in the diagnosis of esophageal squamous-cell carcinoma: A multicenter ex vivo pilot study. Gastrointest Endosc 2007;66:551-555.

13. Kumagai Y, Kawada K, Yamazaki S, et al. Endocytoscopic observation for esophageal squamous cell carcinoma: can biopsy histology be omitted? Dis Esophagus 2009;22:505-512.

14. Minami $\mathrm{H}$, Inoue $\mathrm{H}$, Yokoyama $\mathrm{A}$, et al. Recent advancement of observing living cells in the esophagus using CM double staining: endocytoscopic atypia classification. Dis Esophagus 2012;25:235-241.

15. Kaise M, Kimura R, Nomura K, et al. Accuracy and concordance of endocytoscopic atypia for the diagnosis of gastric cancer. Endoscopy 2014;46:827-832.

16. Kaise M, Ohkura Y, Iizuka T, et al. Endocytoscopy is a promising modality with high diagnostic accuracy for gastric cancer. Endoscopy 2015;47:19-25.

17. Sato $\mathrm{H}$, Inoue $\mathrm{H}$, Hayee $\mathrm{B}$, et al. In vivo histopathology using endocytoscopy for non-neoplastic changes in the gastric mucosa: a prospective pilot study (with video). Gastrointest Endosc 2015; 81:875-881.

18. Sasajima $\mathrm{K}$, Kudo SE, Inoue $\mathrm{H}$, et al. Real-time in vivo virtual histology of colorectal lesions when using the endocytoscopy system. Gastrointest Endosc 2006;63:1010-1017.

19. Kudo SE, Wakamura K, Ikehara N, Mori Y, Inoue H, Hamatani S. Diagnosis of colorectal lesions with a novel endocytoscopic 
classification - a pilot study. Endoscopy 2011;43:869-875.

20. Pohl H, Rösch T, Tanczos BT, Rudolph B, Schlüns K, Baumgart DC. Endocytoscopy for the detection of microstructural features in adult patients with celiac sprue: a prospective, blinded endocytoscopyconventional histology correlation study. Gastrointest Endosc 2009;70:933-941.

21. Matysiak-Budnik T, Coron E, Mosnier JF, Le Rhun M, Inoue H, Galmiche JP. In vivo real-time imaging of human duodenal mucosal structures in celiac disease using endocytoscopy. Endoscopy 2010;42:191-196.

22. Kodashima S, Fujishiro M, Takubo K, et al. Ex-vivo study of highmagnification chromoendoscopy in the gastrointestinal tract to determine the optimal staining conditions for endocytoscopy. Endoscopy 2006;38:1115-1121.

23. Ichimasa K, Kudo SE, Mori Y, et al. Double staining with crystal violet and methylene blue is appropriate for colonic endocytoscopy: an in vivo prospective pilot study. Dig Endosc 2014;26:403-408.

24. Kumagai Y, Kawada K, Yamazaki S, et al. Current status and limitations of the newly developed endocytoscope GIF-Y0002 with reference to its diagnostic performance for common esophageal lesions. J Dig Dis 2012;13:393-400.

25. Schlemper RJ, Riddell RH, Kato Y, et al. The Vienna classification of gastrointestinal epithelial neoplasia. Gut 2000;47:251-255.

26. Olliver JR, Wild CP, Sahay P, Dexter S, Hardie LJ. Chromoendoscopy with methylene blue and associated DNA damage in Barrett's oesophagus. Lancet 2003;362:373-374.

27. Kiesslich R, Burg J, Vieth M, et al. Confocal laser endoscopy for diagnosing intraepithelial neoplasias and colorectal cancer in vivo. Gastroenterology 2004;127:706-713.

28. Kantsevoy SV, Adler DG, Conway JD, et al. ASGE Technology Committee. Confocal laser endomicroscopy. Gastrointest Endosc 2009;70:197-200.

29. Mori Y, Kudo S, Ikehara N, et al. Comprehensive diagnostic ability of endocytoscopy compared with biopsy for colorectal neoplasms: a prospective randomized noninferiority trial. Endoscopy 2013; 45:98-105. 